\title{
Administration of Ticagrelor and Double-Dose Clopidogrel Based on Platelet Reactivity Determined by VerifyNow-P2Y12 for Chinese Subjects After Elective PCI
}

\author{
Xi Wu, ${ }^{1 *} \mathrm{MD}$, Gang Liu, ${ }^{2 *} \mathrm{MD}$, Jie Lu, ${ }^{1} \mathrm{MD}$, Xin-Xin Zheng, ${ }^{1} \mathrm{MD}$, \\ Jin-Gang CuI, ${ }^{1} \mathrm{MD}$, Xue-Yan ZhaO, ${ }^{1} \mathrm{MD}$, and Xiao-Hong Huang, ${ }^{1} \mathrm{MD}$
}

\begin{abstract}
SUMMARY
Previous studies have identified high on treatment platelet reactivity (HTPR) as a potent factor predicting ischemic events for patients with coronary heart disease. We assessed the efficacy and safety of ticagrelor (90 mg twice-daily) and double-dose of clopidogrel (150 mg once-daily) among Chinese patients for elective percutaneous coronary intervention. We enrolled 40 patients with HTPR from among 317 patients with non-ST-segment elevation acute coronary syndromes after a successful elective percutaneous coronary intervention (PCI). Platelet reactivity was measured by VerifyNow P2Y12 assay. Platelet reactivity was significantly lower for both groups when compared with baseline platelet reactivity after medication adjustment (all $P<0.001$ ). The mean platelet reactivity units (PRU) was significantly lower for the ticagrelor group compared with that of the clopidogrel group over time (all $P<0.001$ ). The differences in the rate of sustained HTPR at different time points between the two groups were significant ( 2 hours: $0 \%$ versus $60 \%$; 8 hours: $5.6 \%$ versus 50\%; 24 hours: $5.9 \%$ versus $43.8 \%$, all $P<0.05$ ). Genetic variation of CYP2C19*2 had no impact on PRU means or rate of HTPR in the ticagrelor group $(P>0.05)$. During the 30-day follow-up, no MACE occurred in any patient, and the overall risk of bleeding showed no difference between the two groups ( $35 \%$ versus $21 \%, P=0.48)$. Our results suggest that ticagrelor may achieve a more rapid and greater platelet inhibition than double-dose clopidogrel. Further studies are still needed to assess the differences in efficacy and safety between ticagrelor and double-dose clopidogrel administration for Chinese patients post elective PCI. (Int Heart J 2017; 58: 167-173)
\end{abstract}

Key words: High platelet reactivity, CYP2C19

$\mathrm{P}$ latelet activation is a key factor that results in stent thrombosis after percutaneous coronary intervention (PCI). ${ }^{1)}$ Dual antiplatelet therapy with aspirin and clopidogrel is the standard treatment for patients after drugeluting stent implantation for coronary disease. However, clinical responses to clopidogrel are highly variable between patients, due to the existence of high platelet reactivity (HPR), and some patients on standard dual antiplatelet therapy still have adverse events after acute coronary syndromes (ACS) and $\mathrm{PCI}^{2-4)}$ On this occasion, raising the dose of clopidogrel or changing to more potent antiplatelet drugs may be a solution for clopidogrel resistance and to improve clinical outcome.

Ticagrelor, namely cyclopentyltriazolopyrimidine, is a new orally administered direct-acting P2Y12-receptor antagonist. ${ }^{5)}$ Ticagrelor binds reversibly and noncompetitively to the P2Y12 receptor, while the thienopyridine compounds clopidogrel and prasugrel bind irreversibly to the P2Y12 receptor, and has distinct pharmacologic properties. ${ }^{6}$ In the Platelet Inhibition and Patient Outcomes (PLATO) trial, ticagrelor was associated with a significant risk reduction in the primary composite endpoint of vascular death, nonfatal MI, or nonfatal stroke compared with clopidogrel in patients with acute coronary syndrome. ${ }^{7}$ However, platelet responsiveness to ticagrelor treatment may vary among patients with different clinical and pathologic features. Few studies on the pharmacodynamics and outcomes of ticagrelor administration guided by platelet function tests have been conducted in Chinese subjects. In fact, the proportion of prescriptions for ticagrelor is relatively small since clinical data is still inconclusive for Chinese.

Therefore, the aims of this study were to explore the efficacy and safety of the combined dosage of clopidogrel with ticagrelor in patients undergoing elective PCI and exhibiting HPR determined by VerifyNow P2Y12 assay, and then to explore the possible association of the antiplatelet response with CYP2C19*2 genotype.

From the ${ }^{1}$ Department of Cardiology, Fuwai Hospital, National Center for Cardiovascular Diseases, Chinese Academy of Medical Science and Peking Union Medical College, Beijing and ${ }^{2}$ Department of Cardiology, The First Affiliated Hospital, Sun Yat-Sen University, Guangzhou, China.

These authors contributed equally to this work.

This study was supported by a grant from the Science and Technology Project of the Beijing Municipal Science and Technology Commission (2013-BKJ10 to Huang Xiao-Hong).

Address for correspondence: Xiao-Hong Huang, MD, Department of Cardiology, Fuwai Hospital, National Center for Cardiovascular Diseases, Chinese Academy of Medical Science and Peking Union Medical College, 167 BeiLiShi Road, Xichen District, Beijing 100037, China. E-mail: huangxh_fuwai@163.com

Received for publication May 7, 2016. Revised and accepted July 19, 2016.

Released in advance online on J-STAGE March 17, 2017.

All rights reserved by the International Heart Journal Association. 


\section{METHODS}

Study design and participants: This study was a randomized, open label study carried out at Fuwai Hospital that enrolled consecutive patients undergoing elective PCI between April 2014 and April 2015. The study protocol conforms to the principles of the Declaration of Helsinki, and was approved by the Ethics Committee of Fuwai Hospital. Patients gave written informed consent prior to study participation. All subjects were under a clopidogrel regimen $(75 \mathrm{mg} /$ day $)$ for at least 1 week before planned coronary angiography and PCI according to current guidelines. First, the residual platelet reactivity of patients with non-ST-elevation acute coronary syndrome were measured with a VerifyNow P2Y12 assay (Accumetrics, San Diego, CA) in peripheral blood samples. Patients presenting with values exceeding 235 PRU were defined as high on treatment platelet reactivity (HTPR). Finally, 40 patients with HTPR enrolled were randomized to a ticagrelor group $(n=20)$ or a clopidogrel group $(n=20)$. The ticagrelor group was administered as a loading dose of $180 \mathrm{mg}$ followed by $90 \mathrm{mg}$ twice daily, while the clopidogrel group was followed by 150 $\mathrm{mg}$ daily of maintenance therapy for 30 days before changing to the normal dosage. All patients received background aspirin therapy at a dose of $100 \mathrm{mg}$ per day. One patient in the double dose clopidogrel group was lost to follow-up.

Key inclusion criteria were age $>18$ years old, patients with a successful elective PCI, and defined as high on treatment platelet reactivity by platelet function test. Exclusion criteria: severe sinus bradycardia, unstable hemodynamics, chronic obstructive pulmonary disease or asthma, history of stroke or transient ischemic attack, long-term oral anticoagulant administration, recent major trauma or surgery, gastrointestinal bleeding or active peptic ulceration, platelet count < $100 \times 10^{9} / \mathrm{L}$ at the time of screening, intracranial neoplasm, arteriovenous malformation or aneurysm, severe renal failure or $\mathrm{CrCl}<25 \mathrm{~mL} /$ minute, severe hypohepatia, and taking or intention to use a potent inhibitor of CYP3A4 such as clarithromycin, ketoconazole, nefazodone, ritonavir or atazanavir. A flow diagram of the study and randomization process is shown in Figure 1.

Platelet function test: In the morning after drug-eluting stent implantation, the PRU values at baseline and 2 hours, 8 hours, and 24 hours after the administration of $150 \mathrm{mg}$ clopidogrel and $180 \mathrm{mg}$ ticagrelor were measured. Five $\mathrm{mL}$ of fasting venous blood was collected into a vacutainer containing 3.2\% sodium citrate and the initial $2 \mathrm{~mL}$ of blood drawn was discarded to avoid measuring platelet activation induced by needle puncture. The Verify Now system is a turbidimetry-based optical detection device that measures platelet induced aggregation in a system containing fibrinogen-coated beads. In the cartridge used for this assay, there is a channel in which inhibition of the P2Y12 receptor is measured; this channel contains ADP as a platelet agonist and prostaglandin E1 as a suppressor of intracellular free calcium levels, to reduce the nonspecific contribution of ADP binding to P2Y12 receptors. Reported measures included PRU and P2Y12 device-reported percent inhibition. Residual platelet reactivity is expressed as PRU. Patients presenting with a PRU value of $\geq 235$ are defined as high on treatment platelet reactivity (HTPR) according to previous studies. $^{8,9)}$

CYP2C19*2 genotyping: Restriction fragment length poly-

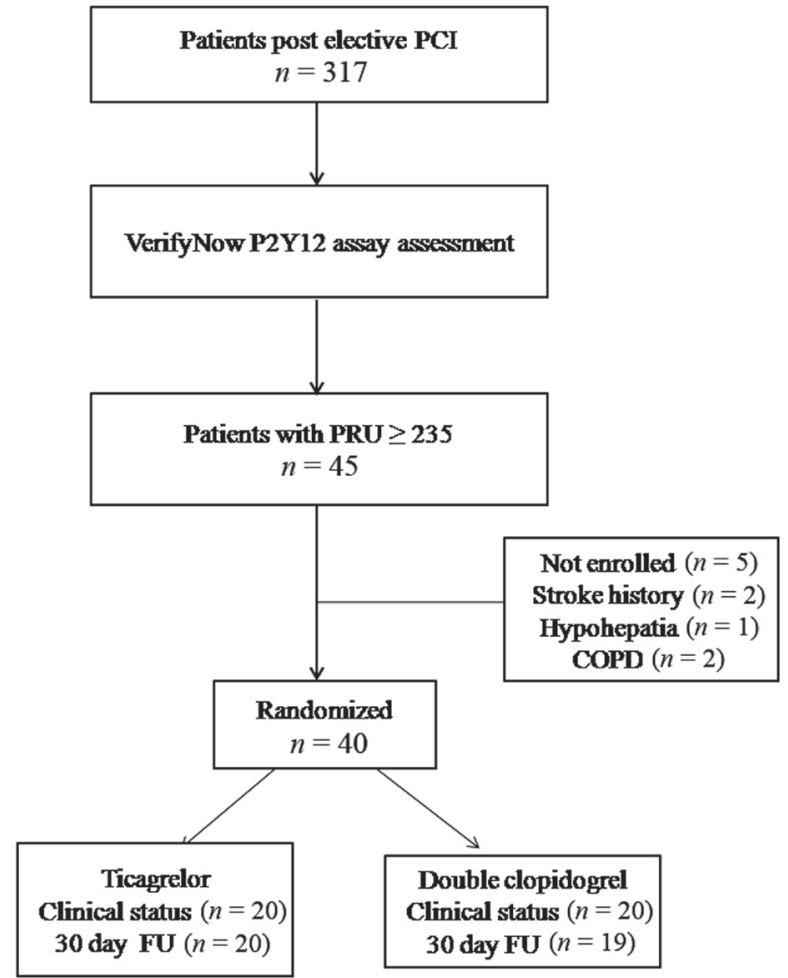

Figure 1. Study design. PRU indicates platelet reactivity units, COPD, chronic obstructive pulmonary disease; and FU, follow-up.

morphism was used to detect genotypes of CYP2C19. DNA was extracted from peripheral blood lymphocytes by standard procedures. Genotyping for known variants *2 of the CYP2C19 gene was performed by PCR amplification. DNA fragmentation from amplification was digested by restriction endonuclease SamI, and restricted DNA products were then separated by cataphoresis. Genotyping was decided by numbers and location of strip. Primers were as follows: Forward, 5'-AGCAGGTATAAGTCTAGGAAA-3', Reverse, 5'-ACAA ATACGCAAGCAGT C-3'.

Follow-up and definitions: Clinical follow-up via telephone contact was scheduled for all patients at 30 days after medication adjustment. Major adverse cardiac events and safety (mainly hemorrhage and dyspnea) of ticagrelor and double dose of clopidogrel were evaluated. Major adverse cardiac events (MACE) were defined as cardiovascular death, acute coronary syndrome, stroke, stent thrombosis, and target vessel revascularization. Cardiac death was defined as death from cardiogenic shock, ventricular arrhythmia (ventricular tachycardia or fibrillation), and coronary artery disease. Hemorrhage was assessed according to the Thrombolysis in Myocardial Infarction (TIMI) bleeding criteria previously described ${ }^{10,11)}: 1$ ) major bleeding: any intracranial bleeding or clinically overt signs of hemorrhage (including imaging diagnosis), associated with a drop in hemoglobin of $\geq 5 \mathrm{~g} / \mathrm{dL}$; 2) minor bleeding: clinically overt (including imaging diagnosis), resulting in hemoglobin drop 3-5 g/dL; 3) minimal bleeding: any overt bleeding event that does not meet the criteria above, resulting in hemoglobin drop $<3 \mathrm{~g} / \mathrm{dL}$.

Statistical analysis: Continuous data with Gaussian distribu- 
tion are expressed as the mean \pm SEM, while abnormal distribution data are reported as the median (P25, P75). Categorical data are expressed as frequencies and percentages (\%). Statistical comparisons were performed with the Mann-Whitney $U$ test, the paired and unpaired Student $t$-test, and the chi-square or Fisher exact test. Analysis of the difference in PRU between ticagrelor and clopidogrel at different time points after administration was performed using a mixed-effect model of repeated measures two-way ANOVA with fixed effects for treatment and period. Comparison of the intra-group difference in PRU at different time points was performed with the paired Student $t$-test and followed by the Bonferroni test. $P<0.05$ was taken to indicate statistical significance, and all statistical analyses were performed using SPSS 21.0 software.

\section{RESUlts}

Patient characteristics: Of 317 patients after a successful nonurgent percutaneous coronary intervention, 45 were defined as HTPR by VerifyNow P2Y12 assay. However, 5 patients met the exclusion criteria, and the other 40 patients were randomized 1:1 to the 2 groups. The clinical characteristics and demographics of the randomised subjects are shown in Table I.
The mean age was $60.4 \pm 10.7$ years in the clopidogrel group versus $62.7 \pm 10.6$ years in the ticagrelor group $(P=0.54)$. Other baseline characteristics, such as sex, comorbidities, laboratory tests, risk factors, drugs, procedural characteristics, and baseline platelet function were similar in both groups.

Comparison of on-treatment residual platelet activity: PRU according to treatment and time are depicted in Table II. Overall, on-treatment platelet reactivity was significantly lower for both groups when compared with baseline platelet reactivity at different time points $(F=59.1, P<0.001)$. The PRU means of ticagrelor descended rapidly at 2 hours, and the interaction between treatment effects and time effects was significant $(F=$ $33.5, P<0.001)$, which indicated that the decrease in amplitude of PRU in the ticagrelor group over time was larger than than that of the clopidogrel group. The main effect of treatment was significant since the mean PRU was significantly lower for the ticagrelor group compared with that of the clopidogrel group at 2 hours, 8 hours, and 24 hours, respectively (all $P<$ 0.001 , Figure $2 \mathrm{~A}$ ). However, the results of multiple comparison tests indicated that the differences in the PRU means between two adjacent time points were not significant in either the clopidogrel group or ticagrelor group.

Rate of HTPR along time: All subjects showed PRU $<235$ at 2 hours after administration of ticagrelor, but one patient at 8

Table I. Baseline Characteristics of Patients

\begin{tabular}{|c|c|c|c|}
\hline Variable & $\begin{array}{l}\text { Ticagrelor group } \\
\quad(n=20)\end{array}$ & $\begin{array}{l}\text { Clopidogrel group } \\
\quad(n=20)\end{array}$ & $P$ \\
\hline Age (years) & $60.4 \pm 10.7$ & $62.7 \pm 10.6$ & 0.54 \\
\hline Male, $n(\%)$ & $15(75)$ & $14(70)$ & 0.59 \\
\hline Number of DES & $1.7 \pm 0.7$ & $1.8 \pm 0.9$ & 0.86 \\
\hline \multicolumn{4}{|l|}{ Risk factor } \\
\hline Body weight (kg) & $68.5 \pm 3.8$ & $66.7 \pm 3.9$ & 0.74 \\
\hline $\operatorname{BMI}\left(\mathrm{kg} / \mathrm{m}^{2}\right)$ & $26.1 \pm 3.8$ & $26.2 \pm 3.7$ & 0.94 \\
\hline Smoking & $11(55)$ & $6(30)$ & 0.11 \\
\hline Hypertension & $10(50)$ & $14(70)$ & 0.22 \\
\hline Diabetes & $9(45)$ & $6(30)$ & 0.39 \\
\hline Hyperlipidemia & $16(80)$ & $12(60)$ & 0.17 \\
\hline Prior MI & $2(10)$ & $1(5)$ & 0.42 \\
\hline Prior PCI or CABG & $6(30)$ & $5(25)$ & 0.72 \\
\hline Peripheral vascular diseases & $15(75)$ & $14(70)$ & 0.74 \\
\hline $\operatorname{LVEF}(\%)$ & $63.7 \pm 5.8$ & $62.9 \pm 8.4$ & 0.73 \\
\hline \multicolumn{4}{|l|}{ Laboratory data } \\
\hline $\mathrm{RBC}\left(* 1000 / \mathrm{mm}^{3}\right)$ & $4.4 \pm 0.4$ & $4.2 \pm 0.5$ & 0.12 \\
\hline $\mathrm{WBC}\left(* 1000 / \mathrm{mm}^{3}\right)$ & $6.9 \pm 1.4$ & $6.8 \pm 1.6$ & 0.73 \\
\hline Platelets $\left(* 1000 / \mathrm{mm}^{3}\right)$ & $207 \pm 40.3$ & $209.9 \pm 58.7$ & 0.87 \\
\hline hs-CRP, mg/L & $4.6 \pm 3.8$ & $3.4 \pm 3.2$ & 0.34 \\
\hline Creatinine $(\mu \mathrm{mol} / \mathrm{L})$ & $74.8 \pm 15.6$ & $72.2 \pm 15.9$ & 0.62 \\
\hline $\mathrm{cTnI}(\mathrm{ng} / \mathrm{mL})$ & $0.27 \pm 0.5$ & $0.19 \pm 0.4$ & 0.59 \\
\hline CK-MB (ng/mL) & $2.6 \pm 1.9$ & $2.9 \pm 1.6$ & 0.61 \\
\hline \multicolumn{4}{|l|}{ Medication (\%) } \\
\hline Beta-blockers & $20(100)$ & $17(85)$ & 0.11 \\
\hline Calcium-channel blockers & $12(60)$ & $10(50)$ & 0.45 \\
\hline ACEI & $3(15)$ & $5(25)$ & 0.35 \\
\hline $\mathrm{ARB}$ & $10(50)$ & $8(40)$ & 0.55 \\
\hline Statin & $20(100)$ & $20(100)$ & 1.0 \\
\hline PPI & $12(60 \%)$ & $14(70 \%)$ & 0.73 \\
\hline CYP2C19*2 carriers $(\%)$ & $9(45 \%)$ & $7(35 \%)$ & 0.52 \\
\hline \multicolumn{4}{|l|}{ Baseline platelet reactivity } \\
\hline VerifyNow PRU & $263.2 \pm 6.5$ & $274.8 \pm 7.7$ & 0.26 \\
\hline VerifyNow BASE & $261.6 \pm 9.1$ & $269.9 \pm 8.9$ & 0.51 \\
\hline
\end{tabular}

DES indicates drug eluting stent; MI, myocardial infarction; PCI, percutaneous coronary intervention; CABG, coronary artery bypass grafting; RBC, red blood cell; WBC, white blood cell; ACEI, angiotensin converting-enzyme inhibitor; ARB, angiotensin-receptor II antagonist; PPI, proton pump inhibitor; and PRU, platelet reactivity units. 
Table II. Platelet Reactivity According to Treatment and Time

\begin{tabular}{lrrrrrr}
\hline & \multicolumn{2}{c}{ PRU } & & $F$ & $P$ \\
& Basal & 2 hours & 8 hours & 24 hours & \\
\hline Ticagrelor & $263.2 \pm 6.5$ & $72.7 \pm 13.4^{* \dagger}$ & $68.4 \pm 14.8^{* \dagger}$ & $76.8 \pm 14.7^{* \dagger}$ & 60.2 & $<0.001$ \\
Clopidogrel & $274.8 \pm 7.7$ & $240.3 \pm 9.9^{*}$ & $245.3 \pm 9.4^{*}$ & $239.3 \pm 11.2^{*}$ & 3.3 & 0.026 \\
$P$ & 0.258 & $<0.001$ & $<0.001$ & $<0.001$ & \\
\hline
\end{tabular}

Data are expressed as mean \pm SEM. ${ }^{*}$ Represents PRU value compared with baseline at different time point, $P<0.001$ (Bonferroni adjustment); ${ }^{\dagger}$ Represents PRU value of ticagrelor group compared with that of clopidogrel group at each time point, $P<0.001$.

A

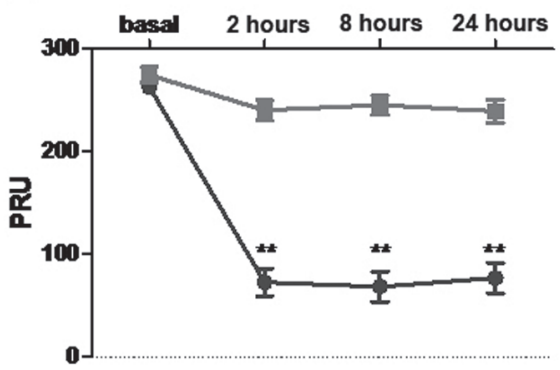

Ticagrelor - Clopidogrel

B

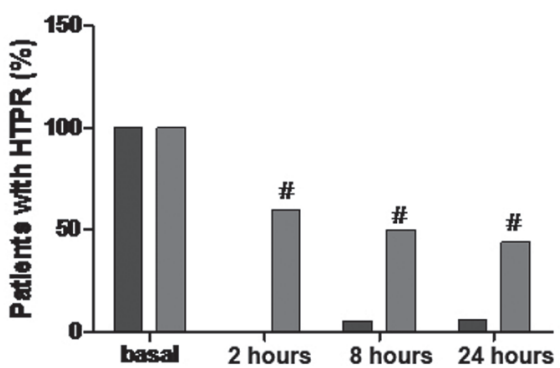

Figure 2. Comparison of mean platelet reactivity and rate of sustained HTPR in the 2 study groups. A: PRU value at different times after medication administration the morning after PCI. ${ }^{* *} P<0.001$. B: Patients with HTPR within 24 hours after medication adjustment. ${ }^{*} P<0.05$.

hours and one patient at 24 hours were identified with HTPR. For the clopidogrel group, the percentages of patients with HTPR at 2 hours, 8 hours, and 24 hours after administration were $60 \%, 50 \%$, and $43.75 \%$, respectively. The differences in the rate of HTPR at different time points between the two groups were significant (all $P<0.05$, Figure 2B).

Association between CYP2C19*2 genotype and antiplatelet effects of ticagrelor: The results of genotyping showed that 9 patients in the ticagrelor group carry at least one afunctional CYP2C19*2 homozygote variant. However, the PRU means were significantly lower for the ticagrelor group compared with that of the clopidogrel group regardless of whether the patients in the ticagrelor group carried the genetic variation or not $(P<0.001$, Figure $3 \mathrm{~A}$ and B). From the results of the present study, genetic variation leading to lack of function of CYP2C19*2 had no impact on PRU means or incidence of HTPR of patients in the ticagrelor group $(P>0.05$, Figure $3 \mathrm{C}$ and D).

Outcome and adverse events: Until day 30, no patient in either treatment group exhibited a major adverse cardiovascular event, and there were no severe side effects like major bleeding leading to study drug discontinuation (Table III). Six patients $(30 \%)$ on ticagrelor reported minimal bleeding events (eg, skin ecchymosis) and one patient (5\%) reported a minor bleeding event (eg, minor hemorrhage of digestive tract). On the other hand, 4 patients $(21 \%)$ receiving double dose clopidogrel reported minimal bleeding events. However, the difference in terms of the overall risk of bleeding was not significant among the two groups $(P=0.48)$. Five patients in the ticagrelor group reported transient dyspnea events but they had no influence on continuing the treatment.

\section{DiscUSSION}

In this study, residual platelet reactivity decreased significantly for both groups after switching from $75 \mathrm{mg}$ clopidogrel to double dose clopidogrel or ticagrelor. However, patients in the ticagrelor group achieved significantly lower PRU at each time point and a higher number of patients randomized to ticagrelor changed their response status. Next, we combined the results of genotype and found the rate of sustained high platelet reactivity was significantly lower in the ticagrelor group regardless of whether the patients had genetic variation or not. During the 30-day follow-up, no MACE occurred in either treatment group, and the overall risk of bleeding showed no difference between the 2 groups. Prior studies have identified HTPR as a potent factor predicting ischemic events. ${ }^{4,12,13)}$ It seems that second-generation drug-eluting stents and biodegradable polymer drug-eluting stents might reduce stent thrombosis or restenosis. ${ }^{14,15)}$ However, the benefits of platelet function test guided therapy and whether identifying HTPR would led to interventions that reduced the risk of ischemic events are not certain.

Among methods for platelet function testing, the VerifyNow P2Y12 system is a real point-of-care device which requires no sample processing and special skills, and can give results in a short time. ${ }^{16)}$ Most importantly, previous studies have suggested the VerifyNow system was well correlated with the gold standard light transmission aggregometry. ${ }^{17,18)}$ Increasing evidence suggests that high residual platelet reactivity detected by VerifyNow assay predicts clinical outcome and adverse cardiovascular events in patients undergoing percutaneous coronary intervention. ${ }^{8,9,19,20)}$ Nevertheless, few studies have assessed the efficacy and safety of double dose clopidogrel and ticagrelor guided by the VerifyNow P2Y12 system for patients undergoing elective PCI. As reported, the optimal cutoff for the combined endpoint was a residual platelet reactivity $\geq 235-240$ PRU. ${ }^{8,9)}$ In the present study, we identified patients with HTPR by PRU $\geq 235$. In China, considering the cost of 


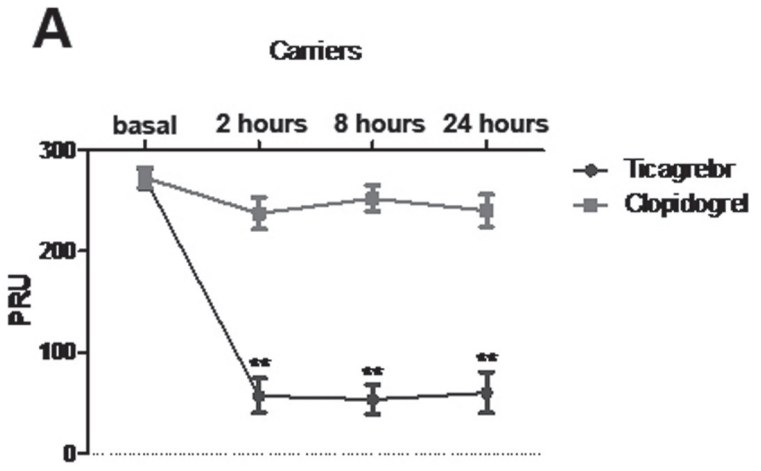

B
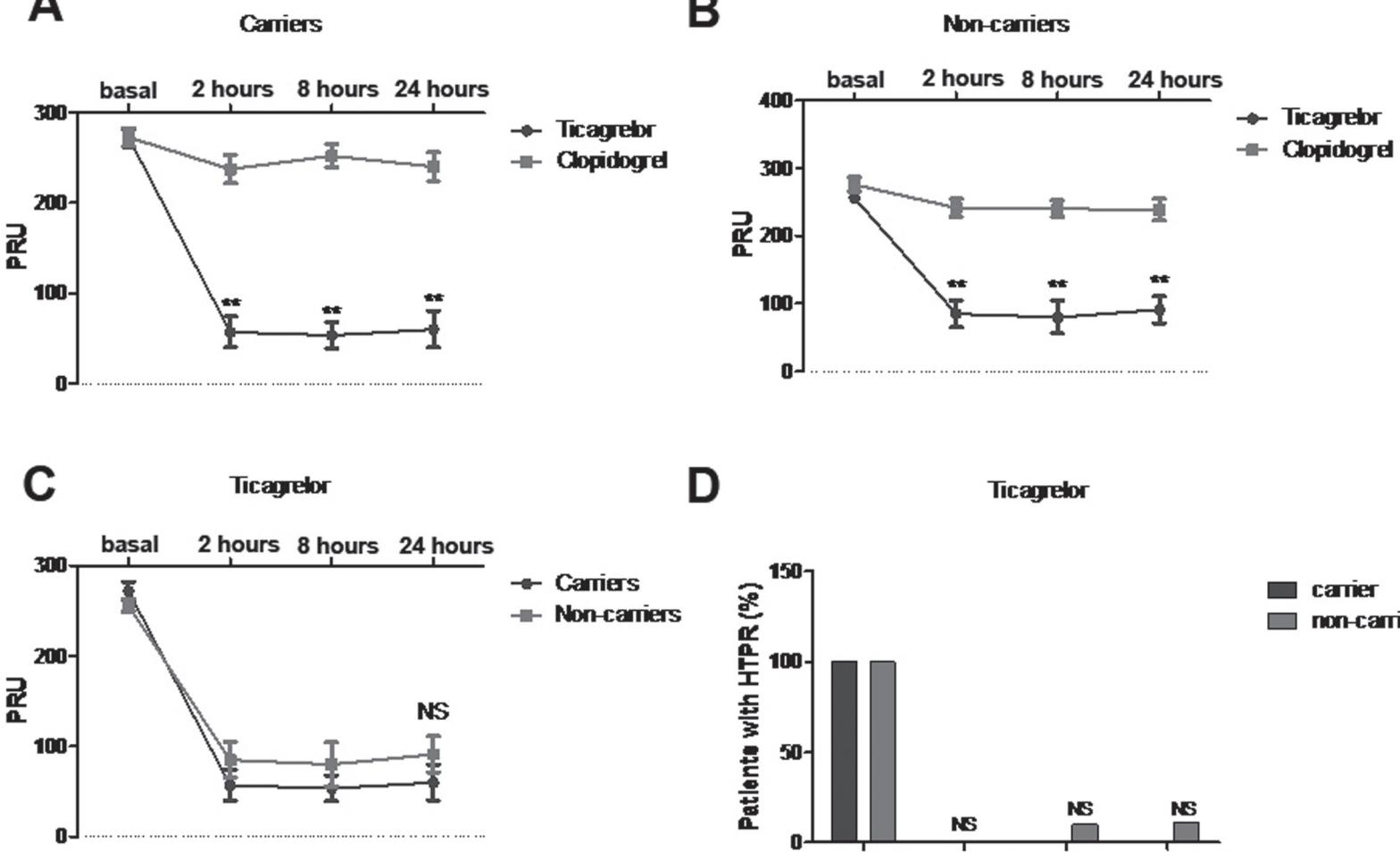

Ticagrebr

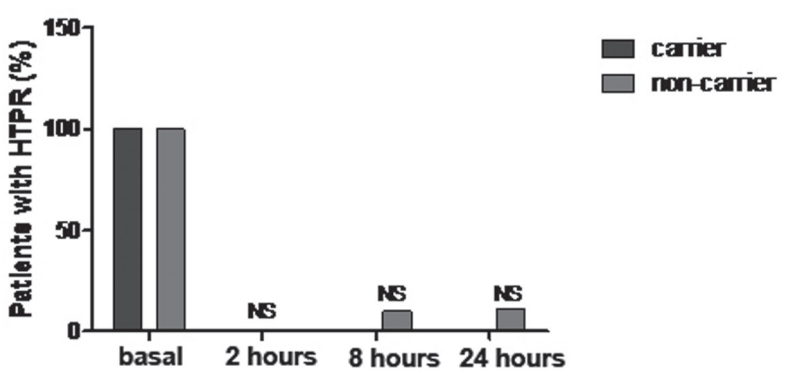

Figure 3. Association between CYP2C19*2 genotype and antiplatelet effects of ticagrelor. A and B: Comparison of antiplatelet effects of ticagrelor and clopidogrel according to CYP2C19*2 genotype. C and D: PRU value and rate of HTPR in patients with or without loss-of-function mutation of CYP2C19*2 in the ticagrelor group.

Table III. Outcome and Adverse Event in 30-Day Follow-Up

\begin{tabular}{lccl}
\hline & $\begin{array}{c}\text { Ticagrelor group } \\
(n=20)\end{array}$ & $\begin{array}{c}\text { Clopidogrel group } \\
(n=19)\end{array}$ & $P$ \\
\hline MACE, $n(\%)$ & $0(0)$ & $0(0)$ & 1.0 \\
Overall bleeds, $n(\%)$ & $7(35)$ & $4(21)$ & 0.48 \\
Major bleeding & $0(0)$ & $0(0)$ & 1.0 \\
Minor bleeding & $1(5)$ & $0(0)$ & 1.0 \\
Minimal bleeding & $6(30)$ & $4(21)$ & 0.79 \\
Dyspnea, $n(\%)$ & $5(25)$ & $0(0)$ & 0.047 \\
\hline
\end{tabular}

MACE indicates major adverse cardiovascular event.

detection and uncertain clinical benefit, the VerifyNow assay is not used routinely in clinical practice. Further studies are still needed to assess the relationship between platelet function and outcomes in order to support the use of VerifyNow P2Y12 to optimize individualized anti-platelet treatment.

Ticagrelor is a potent antagonist of the adenosine diphosphate receptor P2Y12, which provides faster, greater, and more consistent P2Y12 inhibition than clopidogrel. ${ }^{5,21)}$ In the PLATO trial, for patients with acute coronary syndrome, the benefit of ticagrelor over clopidogrel was evident within the first 30 days of treatment and continued to increase from day 31 to 1 year, and the rates of death from vascular causes, myocardial infarction, or stroke were significantly reduced without an increase in the rate of overall major bleeding but with an increase in the rate of non procedure-related bleeding. ${ }^{7}$ The RESPOND Study demonstrated that ticagrelor therapy overcomes nonresponsiveness to clopidogrel, and its antiplatelet effect is the same in responders and nonresponders with stable coronary artery disease. ${ }^{22)}$ As measured by multiple platelet function testing including the VerifyNow P2Y12 system, platelet reactivity was below the cut-off points associated with ischemic risk in $98 \%$ to $100 \%$ of patients after ticagrelor therapy versus $44 \%$ to $76 \%$ of patients after clopidogrel therapy. This provides a possible explanation for the benefit of ticagrelor on outcome. Moreover, in ACS patients exhibiting HTPR, platelet reactivity (32.9 PRU versus 101.3 PRU) and HTPR rate (0\% versus $2.4 \%)$ were markedly lower for ticagrelor treatment than with with prasugrel treatment. ${ }^{23)}$ From the results of our study, platelet reactivity and rate of sustained high platelet reactivity were significantly lower for the ticagrelor group compared with that of the clopidogrel group, which was similar to previous studies. Furthermore, our study shows that the antiplatelet effects of ticagrelor were consistent in patients with or without genetic variation of CYP2C19*2. The PLATO trial also suggested that ticagrelor 
reduces the incidence of stent thrombosis compared with clopidogrel in patients with acute coronary syndromes regardless of CYP2C19 genetic status. ${ }^{24)}$ This can be explained in terms of ticagrelor being a direct-acting agent with no need for CYP2C19 enzymes in liver.

In fact, the evidence on the benefit of doubling clopidogrel or ticagrelor has been mixed, and related clinical data are still inconclusive. Doubling the loading and maintenance doses of clopidogrel may lead to better platelet inhibition or even improved clinical outcomes. ${ }^{25,26)}$ According to the CURRENT trial, for patients with ACS referred for an invasive strategy, a double-dose clopidogrel regimen (eg, 600-mg loading dose on day 1 , followed by $150 \mathrm{mg}$ daily for 6 days before returning to the standard dose) reduced stent thrombosis compared to the standard dose regimen. ${ }^{27)}$ Meanwhile, in the GRAVITAS randomized trial, the use of high-dose clopidogrel for 6 months did not reduce the incidence of death from cardiovascular causes, nonfatal myocardial infarction, or stent thrombosis among patients with HTPR after implantation with drug-eluting stents. ${ }^{28)}$ On the other hand, compared with clopidogrel, ticagrelor resulted in a greater prevention of not only first, but also subsequent cardiovascular events. ${ }^{29)}$ However, the claimed mortality benefits of ticagrelor have been challenged based on the fact that significantly more patients on ticagrelor had incomplete vital status follow-up versus clopidogrel, which may lead to an inappropriate conclusion about ticagrelor. ${ }^{30)}$ Ticagrelor is likely to be superior to double clopidogrel in those patients with emergency PCI since ticagrelor can work faster and is more potent at preventing early stent thrombosis. However, this effect may not be the same for patients undergoing elective PCI. In our 30-day follow-up, there was no significant difference in major adverse cardiovascular events between the two groups, which implies ticagrelor and double-dose clopidogrel administration have the same treatment effects. This is consistent with previous research which indicated that double-dose clopidogrel may reduce stent thrombosis. ${ }^{27)}$ Another explanation for the results is that our sample size was relatively small, which limits its power to analyze the difference. In the PLATO study, life-threatening bleeding and fatal bleeding were not significantly different, whereas there was a significant increase in non-CABG related major bleeding in patients receiving ticagrelor. No severe side effects like major bleeding were observed in the present study so ticagrelor and double-dose clopidogrel are relatively safe for Chinese patients.

Study limitations: This study has several limitations. First, it involved a relatively small number of patients and was conducted at a single center. So the study might be underpowered to assess clinical outcomes. Second, we only assessed platelet reactivity in the 24 hours after medication adjustment, without dynamic monitoring of on-treatment residual platelet activity during the follow-up. Third, we were unable to evaluate platelet activity through different platelet function tests, whose results may vary. So far, platelet function testing and genetic testing are not widespread in China because of their high cost, lack of standards, and relative degree of uncertainty. Finally, the present study examined the antiplatelet effects of ticagrelor and doubling of clopidogrel over a short period of time. The long-term benefit and safety of ticagrelor remain to be investigated in future trials.

Conclusion: This study suggests that ticagrelor may achieve a more rapid and greater platelet inhibition than double-dose clopidogrel. However, the differences in the efficacy and safety of ticagrelor and double clopidogrel administration among Chinese patients still need to be evaluated in larger randomized studies.

\section{Disclosure}

The authors do not have any potential conflicts of interest associated with this paper.

\section{REFERENCES}

1. Levine GN, Bates ER, Blankenship JC, et al. 2011 ACCF/AHA/ SCAI Guideline for Percutaneous Coronary Intervention. A report of the American College of Cardiology Foundation/American Heart Association Task Force on Practice Guidelines and the Society for Cardiovascular Angiography and Interventions. J Am Coll Cardiol 2011; 58: e44-122.

2. Matetzky S, Shenkman B, Guetta V, et al. Clopidogrel resistance is associated with increased risk of recurrent atherothrombotic events in patients with acute myocardial infarction. Circulation 2004; 109: 3171-5.

3. Gurbel PA, Bliden KP, Guyer K, et al. Platelet reactivity in patients and recurrent events post-stenting: results of the PREPARE POST-STENTING Study. J Am Coll Cardiol 2005; 46: 1820-6.

4. Bliden KP, DiChiara J, Tantry US, Bassi AK, Chaganti SK, Gurbel PA. Increased risk in patients with high platelet aggregation receiving chronic clopidogrel therapy undergoing percutaneous coronary intervention: is the current antiplatelet therapy adequate? J Am Coll Cardiol 2007; 49: 657-66.

5. Husted S, van Giezen JJ. Ticagrelor: the first reversibly binding oral P2Y12 receptor antagonist. Cardiovasc Ther 2009; 27: 25974.

6. Dobesh PP, Oestreich JH. Ticagrelor: pharmacokinetics, pharmacodynamics, clinical efficacy, and safety. Pharmacotherapy 2014; 34: 1077-90. (Review)

7. Wallentin L, Becker RC, Budaj A, et al. Ticagrelor versus clopidogrel in patients with acute coronary syndromes. N Engl J Med 2009; 361: 1045-57.

8. Marcucci R, Gori AM, Paniccia R, et al. Cardiovascular death and nonfatal myocardial infarction in acute coronary syndrome patients receiving coronary stenting are predicted by residual platelet reactivity to ADP detected by a point-of-care assay: a 12-month follow-up. Circulation 2009; 119: 237-42.

9. Price MJ, Endemann S, Gollapudi RR, et al. Prognostic significance of post-clopidogrel platelet reactivity assessed by a point-ofcare assay on thrombotic events after drug-eluting stent implantation. Eur Heart J 2008; 29: 992-1000.

10. Rao SV, O'Grady K, Pieper KS, et al. Impact of bleeding severity on clinical outcomes among patients with acute coronary syndromes. Am J Cardiol 2005; 96: 1200-6.

11. Bovill EG, Terrin ML, Stump DC, et al. Hemorrhagic events during therapy with recombinant tissue-type plasminogen activator, heparin, and aspirin for acute myocardial infarction. Results of the Thrombolysis in Myocardial Infarction (TIMI), Phase II Trial. Ann Intern Med 1991; 115: 256-65.

12. Matetzky S, Shenkman B, Guetta V, et al. Clopidogrel Resistance Is Associated With Increased Risk of Recurrent Atherothrombotic Events in Patients With Acute Myocardial Infarction. Circulation 2004; 109: 3171-5.

13. Cuisset T, Frere C, Quilici J, et al. High post-treatment platelet reactivity identified low-responders to dual antiplatelet therapy at increased risk of recurrent cardiovascular events after stenting for acute coronary syndrome. J Thromb Haemost 2006; 4: 542-9.

14. Nishio R, Shinke T, Morino Y, et al. Early phase arterial reaction following drug-eluting and bare-metal stent implantation in pa- 
tients with ST-segment elevation myocardial infarction. Int Heart J 2015; 56: 389-94.

15. Zhang J. Stent thrombosis in patients with coronary artery disease treated with biodegradable polymer drug-eluting stents: an update meta-analysis. Int Heart J 2014; 55: 213-8. (Review)

16. Gajda SN, Kołtowski Ł, Tomaniak M. Most recent evidence behind aggregometry and genotyping methods as platelet function testing for tailored anti-platelet treatment among PCI patients. Adv Clin Exp Med 2015; 24: 687-93. (Review)

17. Zhang HZ, Kim MH, Jeong YH. Predictive values of post-clopidogrel platelet reactivity assessed by different platelet function tests on ischemic events in East Asian patients treated with PCI. Platelets 2014; 25: 292-9.

18. Breet NJ, van Werkum JW, Bouman HJ, Kelder JC, Ten Berg JM, Hackeng CM. High on-aspirin platelet reactivity as measured with aggregation-based, cyclooxygenase- 1 inhibition sensitive platelet function tests is associated with the occurrence of atherothrombotic events. J Thromb Haemost 2010; 8: 2140-8.

19. Patti G, Nusca A, Mangiacapra F, Gatto L, D’Ambrosio A, Di Sciascio G. Point-of-care measurement of clopidogrel responsiveness predicts clinical outcome in patients undergoing percutaneous coronary intervention results of the ARMYDA-PRO (Antiplatelet therapy for Reduction of MYocardial Damage during AngioplastyPlatelet Reactivity Predicts Outcome) study. J Am Coll Cardiol 2008; 52: 1128-33.

20. Valgimigli M, Campo G, de Cesare N, et al. Intensifying platelet inhibition with tirofiban in poor responders to aspirin, clopidogrel, or both agents undergoing elective coronary intervention: results from the double-blind, prospective, randomized Tailoring Treatment with Tirofiban in Patients Showing Resistance to Aspirin and/or Resistance to Clopidogrel study. Circulation 2009; 119: 3215-22.

21. VAN Giezen JJ, Nilsson L, Berntsson P, et al. Ticagrelor binds to human P2Y(12) independently from ADP but antagonizes ADPinduced receptor signaling and platelet aggregation. J Thromb Haemost 2009; 7: 1556-65.

22. Gurbel PA, Bliden KP, Butler K, et al. Response to ticagrelor in clopidogrel nonresponders and responders and effect of switching therapies: the RESPOND study. Circulation 2010; 121: 1188-99.

23. Alexopoulos D, Galati A, Xanthopoulou I, et al. Ticagrelor versus prasugrel in acute coronary syndrome patients with high on-clopidogrel platelet reactivity following percutaneous coronary intervention: a pharmacodynamic study. J Am Coll Cardiol 2012; 60: 193-9.

24. Steg PG, Harrington RA, Emanuelsson H, et al. Stent thrombosis with ticagrelor versus clopidogrel in patients with acute coronary syndromes: an analysis from the prospective, randomized PLATO trial. Circulation 2013; 128: 1055-65.

25. Patti G, Colonna G, Pasceri V, Pepe LL, Montinaro A, Di Sciascio G. Randomized trial of high loading dose of clopidogrel for reduction of periprocedural myocardial infarction in patients undergoing coronary intervention: results from the ARMYDA-2 (Antiplatelet therapy for Reduction of MYocardial Damage during Angioplasty) study. Circulation 2005; 111: 2099-106.

26. Angiolillo DJ, Shoemaker SB, Desai B, et al. Randomized comparison of a high clopidogrel maintenance dose in patients with diabetes mellitus and coronary artery disease: results of the Optimizing Antiplatelet Therapy in Diabetes Mellitus (OPTIMUS) study. Circulation 2007; 115: 708-16.

27. CURRENT-OASIS 7 Investigators, Mehta SR, Bassand JP, Chrolavicius $\mathrm{S}$, et al. Dose comparisons of clopidogrel and aspirin in acute coronary syndromes. N Engl J Med 2010; 363: 930-42.

28. Price MJ, Berger PB, Teirstein PS, et al. Standard- vs high-dose clopidogrel based on platelet function testing after percutaneous coronary intervention: the GRAVITAS randomized trial. JAMA 2011; 305: 1097-105.

29. Kohli P, Wallentin L, Reyes E, et al. Reduction in first and recurrent cardiovascular events with ticagrelor compared with clopidogrel in the PLATO Study. Circulation 2013; 127: 673-80.

30. DiNicolantonio JJ, Tomek A. Misrepresentation of vital status follow-up: Challenging the integrity of the PLATO trial and the claimed mortality benefit of ticagrelor versus clopidogrel. Int $\mathbf{J}$ Cardiol 2013; 169: 145-6. 